OPEN

\title{
Transimpedance Matrix (TIM) Measurement for the Detection of Intraoperative Electrode Tip Foldover Using the Slim Modiolar Electrode: A Proof of Concept Study
}

\author{
$* \ddagger$ Tim M. Klabbers, *†Wendy J. Huinck, *‡Floris Heutink, $\dagger$

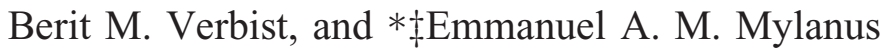

*Department of Otorhinolaryngology and Head and Neck Surgery, Radboud university medical center, Nijmegen, The Netherlands; †Department of Radiology, Leiden University Medical Center, Leiden, The Netherlands; and $\ddagger$ Donders Institute for Brain, Cognition and Behavior, Radboud University, Nijmegen, The Netherlands

Objectives: The aim of this study is to report on our preliminary experience with Transimpedance Matrix (TIM)measurement for the detection of cochlear implant electrode tip foldovers compared with intraoperative imaging in patients implanted with the slim modiolar electrode (SME).

Study Design: Proof of concept study.

Setting: Tertiary university referral center.

Patients: Twenty five ears (in 22 patients) implanted consecutively with the SME.

Intervention(s): Following cochlear implantation, intraoperative TIM-measurement and fluoroscopy were performed. One week postoperatively, the electrode position was evaluated using Computed Tomography (CT)-imaging.

Main Outcome Measures: Electrode array tip foldover.
Results: Electrode array tip foldover occurred in three of the 25 cochlear implantations performed $(12 \%)$. In each case, the foldover was detected by both TIM and fluoroscopy, leading to reposition and correct intracochlear placement of the array.

Conclusions: TIM-measurement is a promising method for the intraoperative detection of an electrode array tip foldover. The TIM-tool with intuitive heatmap display is easy to use, fast, and readily available to clinics using TIM-software in the operating theatre. Key Words: Cochlear implantObjective measures-Tip foldover-Transimpedance matrix.

Otol Neurotol 42:e124-e129, 2021.
The improved understanding of factors influencing residual hearing during cochlear implant (CI) surgery has led to the ability to preserve functional residual hearing in an increasing percentage of CI recipients (1). These factors include surgery-related factors-e.g., the surgical technique used (soft surgery) and the final

Address correspondence and reprint requests to Tim M. Klabbers, M.D., Department of Otorhinolaryngology and Head and Neck Surgery, Radboud university medical center, Philips van Leydenlaan 15, P.O. Box 9101, 6500 HB, Nijmegen, The Netherlands; E-mail: Tim.Klabbers@radboudumc.nl

Source of Funding: Our institute, the department of Otorhinolaryngology and Head \& Neck Surgery of the Radboudumc in the Netherlands, received an ongoing institutional grant from Cochlear Ltd. (Sydney, Australia).

The authors disclose no conflicts of interest.

This is an open access article distributed under the terms of the Creative Commons Attribution-Non Commercial-No Derivatives License 4.0 (CCBY-NC-ND), where it is permissible to download and share the work provided it is properly cited. The work cannot be changed in any way or used commercially without permission from the journal.

DOI: 10.1097/MAO.0000000000002875 intracochlear position of the array (occurrence of scalar translocation or tip foldover), as well as device-related factors-e.g., shape, length, and flexibility of the electrode array $(1-3)$. The implementation of $\mathrm{CI}$ as a whole is aimed at minimizing trauma to the cochlea to reach optimal performance with the device. Present-day cochlear implant arrays can roughly be categorized into two groups: 1) straight, lateral wall (LW) electrodes and 2) precurved, (peri-)modiolar (PM) electrodes. Each design has its benefits and drawbacks. PM electrodes have a reduced intracochlear spread of excitation compared with LW electrodes due to their proximity to the modiolus and therefore lead to the stimulation of a more specific region of spiral ganglion cells, potentially improving speech perception (4). However, a lower rate of full scala tympani (ST) position for the PM electrode compared with the LW electrode (respectively 58 and $89 \%$ ) could, in contrast, lead to lower speech perception scores word scores (5). Therefore, in choosing a perimodiolar electrode, there appears to be a tradeoff between improving intracochlear position and minimizing the risk of insertional trauma. 
A recently introduced electrode array, the slim modiolar electrode (SME), has been designed to be less traumatic while retaining its precurved form and therefore favorable peri-modiolar position. Compared with previous electrode designs of the same manufacturer, the SME is more flexible and thinner, occupying $60 \%$ less volume in the scala tympani. Studies reporting on initial clinical and audiological outcomes confirm its potential for atraumatic insertion (6-8). However, due to its flexibility there could be an increased risk of electrode tip foldover $(6,9)$. Several studies investigating the SME have proven this to be the case, as tip foldover rates using this electrode array vary between 4.3 and $10.5 \%(6,9-$ 12). These rates are distinctly higher than the average tip foldover rate found for both PM electrodes $(1.67 \% ; 13 /$ $778)$ and LW electrodes $(0.23 \% ; 2 / 883)$ in an extensive retrospective case review of 1,772 cochlear surgery reports by Gabrielpillai et al. (10).

Currently, imaging modalities such as (3D rotational) fluoroscopy and plain x-ray imaging are the preferred methods for intraoperative detection of electrode malposition. Studies by Aschendorff et al., McJunkin et al., and Heutink et al. emphasize the importance of intraoperative imaging for SME implantation, but also stress that recognizing a tip foldover on fluoroscopy or plain x-ray imaging can be challenging $(6,9,11)$. In each of these studies, a tip foldover was missed using conventional intraoperative detection methods $(11.1,50$, and $100 \%$ respectively) and was only recognized on postoperative CT-images, resulting in revision surgery $(6,9,11)$.

Other intraoperative investigational methods for evaluating electrode position studied in the past include electrophysiological tests such as electrode impedance measurement, neural response telemetry (NRT), and spread of excitation (SOE) measurements. SOE measurements have been proven to be useful for evaluating electrode position and may play a role in standard cochlear implantation surgery in the future (13). Objective measures modalities such as Electrical Field Imaging (EFI) and Transimpedance Matrix (TIM) measurements, recently termed "SCINSEVs" (Stimulation-CurrentInduced Non-Stimulating Electrode Voltage recordings) by De Rijk et al. (14), have also shown a theoretical potential for evaluating electrode position (15). However, relatively less is known about the clinical value of TIM-measurement in evaluating electrode position, especially tip fold over. The aim of this proof of concept study is to report on our initial experience with TIM-measurement compared with intraoperative imaging for detecting tip foldovers in patients implanted with the SME array.

\section{MATERIALS AND METHODS}

\section{Study Design}

Twenty-two patients with a total of 25 cochlear implantations (three bilateral) were consecutively implanted with the SME (CI532 or CI632, Cochlear Ltd., Sydney, Australia) at the Department of Otorhinolaryngology of the Radboud University medical center, Nijmegen, the Netherlands. One patient (case
24), was preoperatively diagnosed with bilateral enlarged vestibular aqueducts and an incomplete partition type II. All other patients $(n=21)$ had a normal cochlear anatomy. Intraoperative evaluation of correct electrode tip positioning within the cochlea was performed in all cases using both fluoroscopy and TIM-measurements as is standard procedure at our center. In this proof of concept study, the results of these techniques were compared. The study was performed in accordance with the ethical standards of the institutional review board and signed informed consent was obtained for all participants.

\section{Surgical Procedure}

In all patients, access to the cochlea was achieved by performing a standard mastoidectomy and posterior tympanotomy. Three experienced CI surgeons performed the surgeries. Depending on the accessibility of the round window (RW), the surgeon either performed an extended RW approach $(\mathrm{N}=22)$ by removing the crista fenestrae, or a classic cochleostomy anterior and inferior to the round window membrane $(\mathrm{N}=3)$. After loading the SME into the accompanied sheath, the sheath and electrode array were slowly inserted into the cochlea until the sheath stopper of $5 \mathrm{~mm}$ length reached the cochleostomy/ RW membrane opening. Using two forceps, the electrode array was subsequently advanced while holding the sheath in position at the cochleostomy/RW membrane (total insertion time $<120$ s). Following complete insertion, the sheath was retracted and fascia or periosteum with fibrin glue was placed around the array at the cochleostomy/RW membrane opening.

\section{Intraoperative Trans-Impedance Matrix (TIM-) Measurement}

TIM-measurement is an objective measures tool available in CustomSound EP (Version 5.2, Cochlear Ltd., Sydney, Australia) that uses the cochlear implants capability for back-telemetry. During a TIM-measurement, the intracochlear potential profile is measured along each of the 22 electrode contacts while stimulating a single contact in monopolar mode. The voltage measured at the recording electrodes is divided by the injected current at the stimulation contact. When recording and stimulation electrode coincide, the ratio is called the impedance. When they differ, the term trans-impedance is used. In electrode arrays that are correctly positioned in the cochlea, the intracochlear voltage is expected to decrease as the distance between stimulating and recording electrode contacts increases. The result of the TIM-measurement is a matrix of $22 \times 22$ (trans-)impedances. In the software the TIM matrix is colorcoded from high (black and red) to low (green and blue) creating a heatmap. Highest values (black) are recorded at stimulating electrode contacts which can be seen in Figure 1 as a diagonal line from the bottom left corner of the matrix (apical) to the top right (basal). In the event of a tip foldover, the hypothesis is that the spatial distribution of electrode contacts in the cochlea is altered such that apical contacts are located abnormally close to more basal contacts. In the heatmap view, a column contains the voltage (transimpedance) profile along all recording electrodes. When moving away from the stimulation site (diagonal), the values first decrease up to the inflection point of the tip foldover. Beyond this, the values increase again as these apical contacts are physically closer to the stimulation site. This non-monotonic behavior manifests itself as a crosspattern in the heatmap (Fig. 2). The foldover can subsequently be estimated to be at the level of the middle of the "cross" formed by higher (trans-)impedance values (warmer colors) in the heatmap. For all patients included in this study, TIM- 

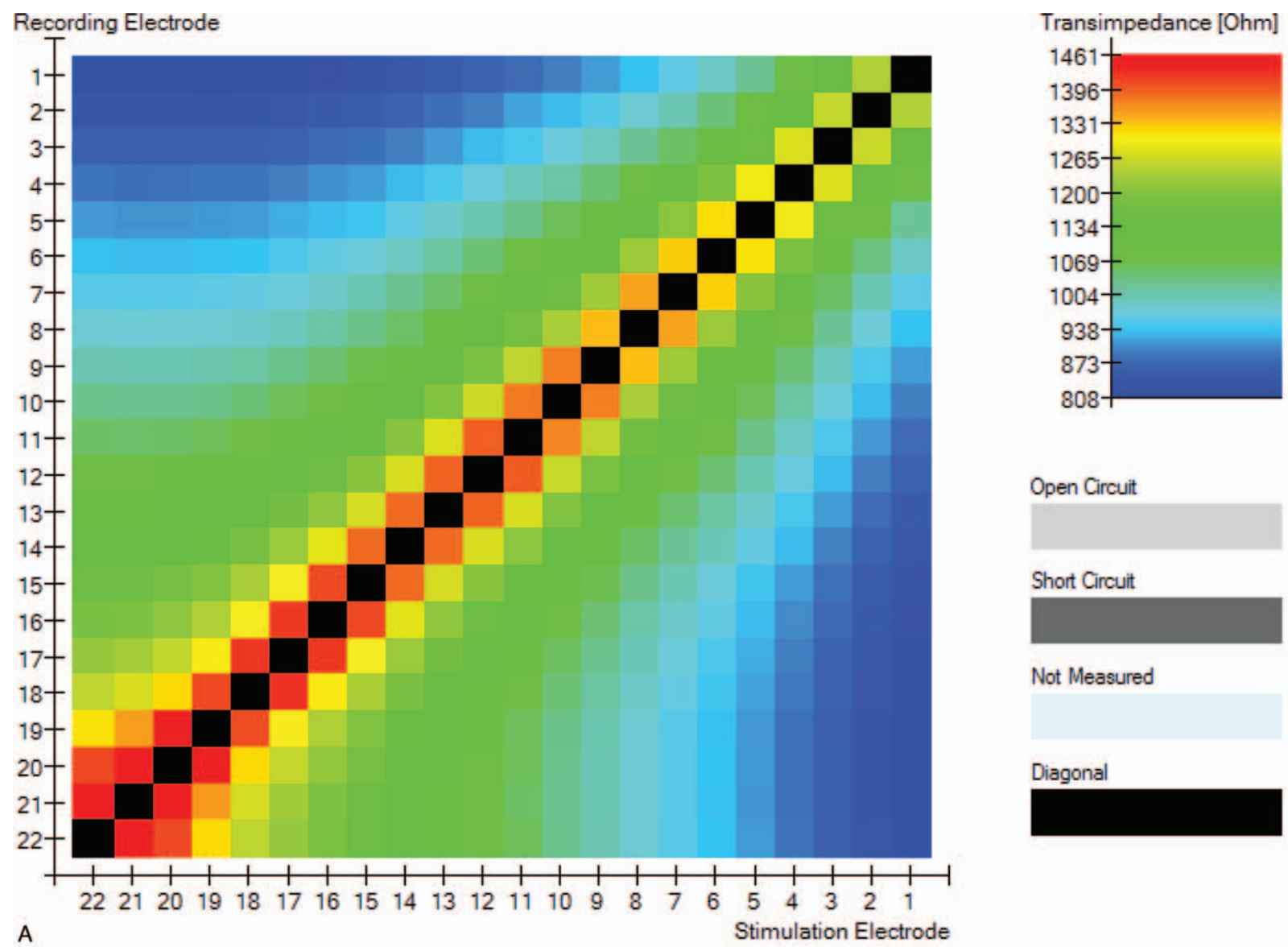

Not Measured

\section{Diagonal}

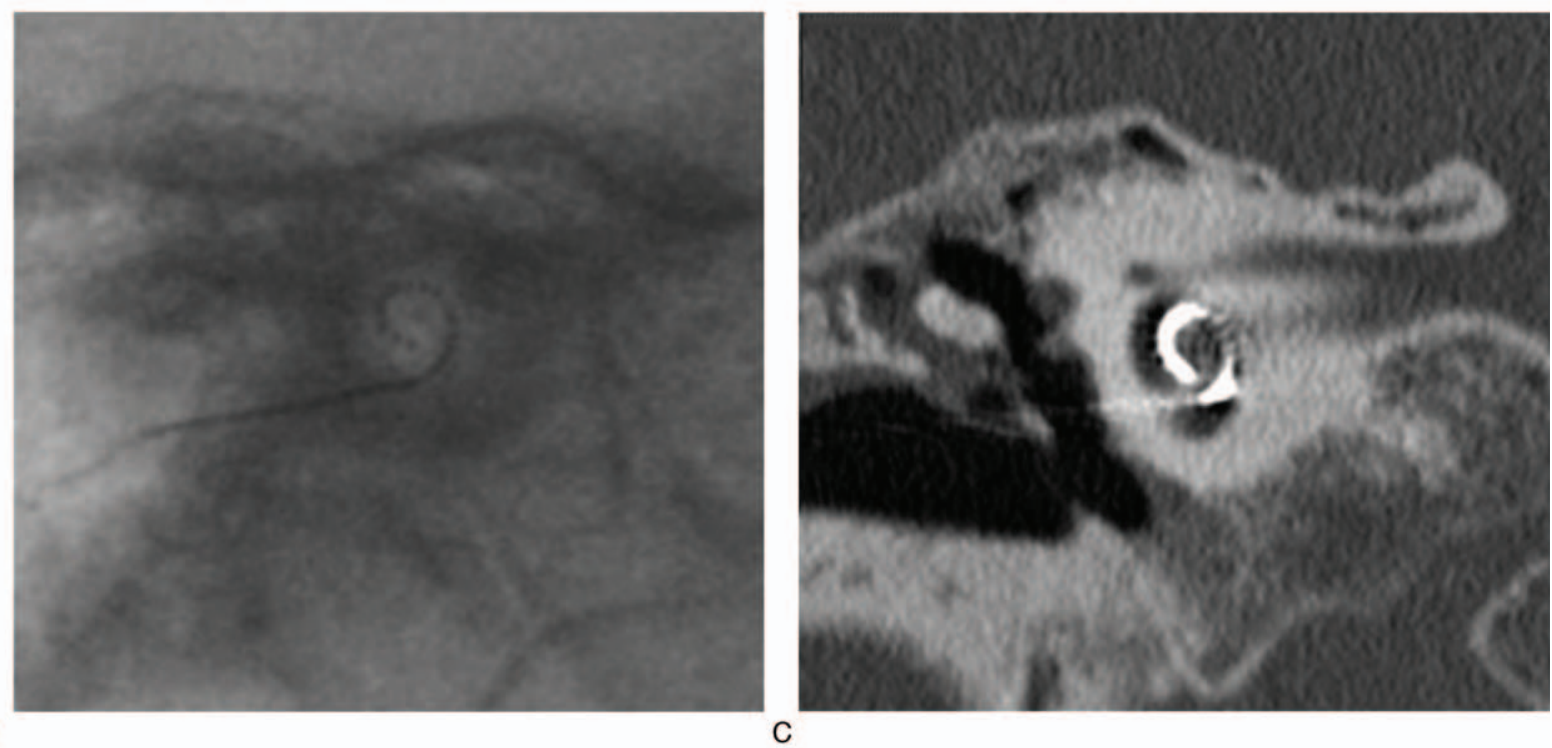

FIG. 1. Intra- and postoperative evaluation of a correctly positioned electrode array for case No. 16. A, Intraoperative TIM-heatmap with accompanying color-legend and corresponding transimpedances (in Ohm) as well as possible error-codes. B, Intraoperative fluoroscopy image (modified Stenver's view). C, Postoperative UHRCT image. TIM indicates Transimpedance Matrix.

measurements were performed in the operating theater using an external sound processor (CP910, Cochlear Ltd., Sydney, Australia) in combination with the TIM-software directly after electrode placement.

\section{Intra- and Postoperative Imaging}

Routine intraoperative fluoroscopy (modified Stenver's view) under sterile conditions was performed in all cases by experienced radiology personnel following TIM-measurement 


\section{Tip Foldover}
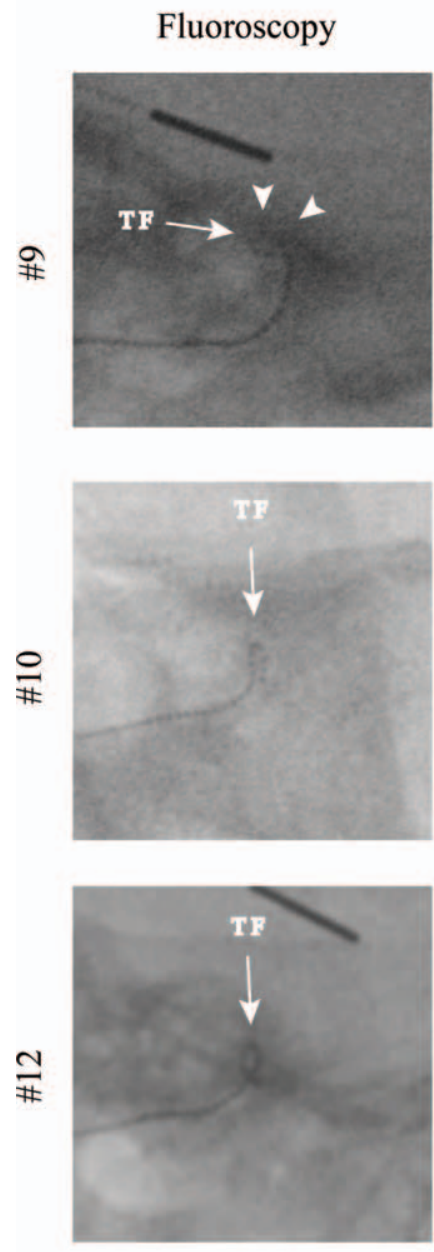

TIM-measurment
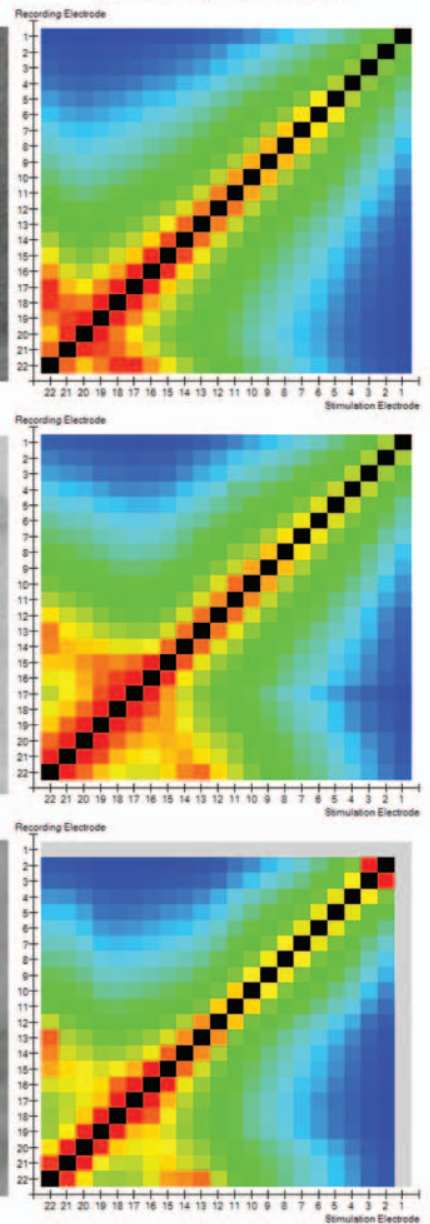

After revision
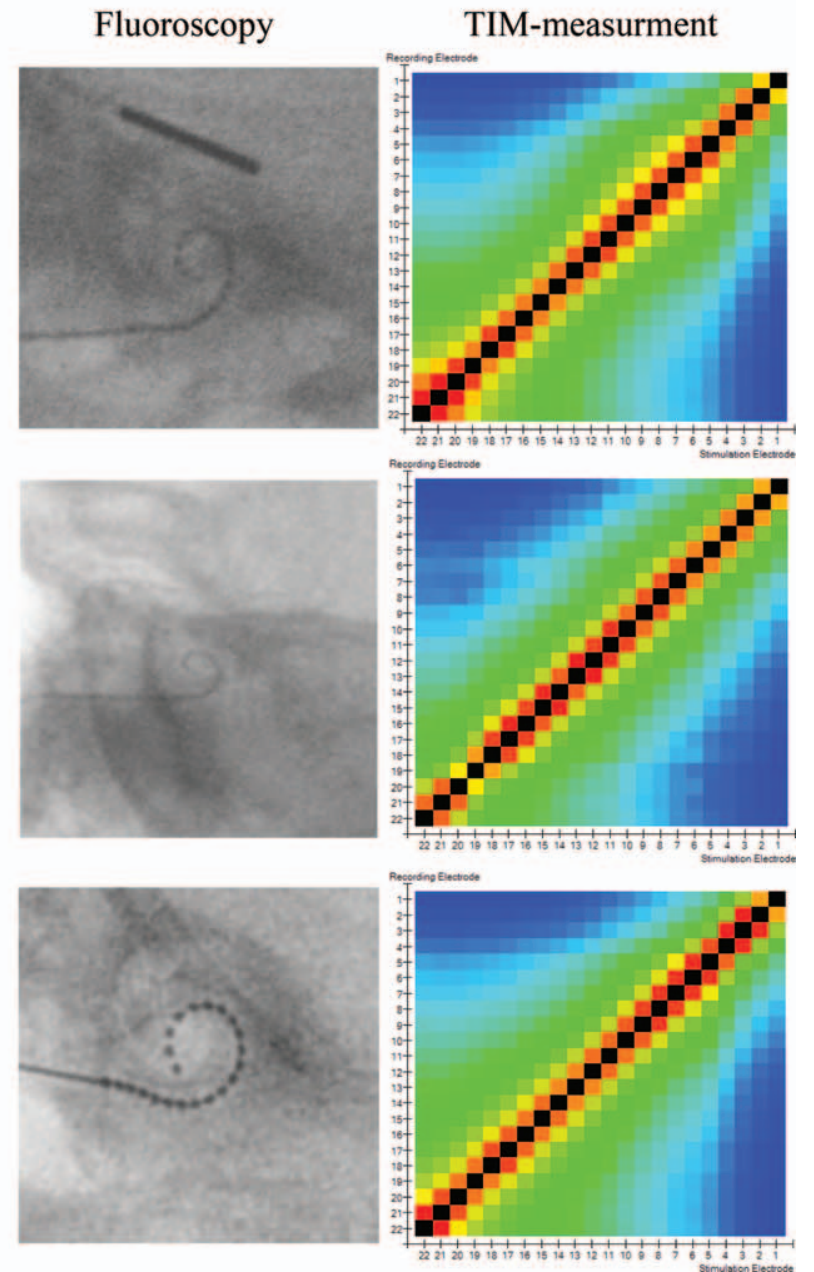

FIG. 2. Intraoperative detection of electrode array tip foldover and evaluation of electrode position after revision surgery using fluoroscopy (modified Stenver's view) and TIM-measurement. The center of the "cross" in tip foldover heatmaps represents the point of inflection. The individual color-legends were left out, as the scale is generated based on the relative differences between (trans)impedances measured along recording contacts. TF indicates tip foldover; TIM, Transimpedance Matrix.

and the images were subsequently evaluated for correct electrode placement by the surgeon before continuing surgery. If a tip foldover was observed, the electrode was removed, reloaded into the sheath and reinserted. After repositioning of the electrode, both fluoroscopy and TIM-measurements were repeated. Electrode positioning was additionally evaluated by an experienced head and neck radiologist 1 week postoperatively using ultra-high resolution computed tomography scanning (UHR computed tomography; Aquilion Precision, Canon Medical Systems, Otawara, Japan) in all adult patients $(\mathrm{N}=17)$. Children $(<18$ yrs, $N=5)$, were not subjected to additional CTscanning to avoid radiation exposure.

\section{RESULTS}

In this proof of concept study, 22 consecutive patients with a total of 25 cochlear implantations (three bilateral) were successfully implanted with the SME. Intraoperative TIM-measurements and fluoroscopy indicated a satisfactory electrode array position in 22 out of 25 cases (Fig. 1). Three tip foldovers were observed intraoperatively $(12 \%)$. Two tip foldovers were detected in children ( $<1$ year old) and one in an adult patient (Table 1). Figure 2 presents the comparison of the fluoroscopy images and TIM-heatmaps for the three tip foldover cases after initial, incorrect placement as well as after reinsertion. In cases Nos 9 and 12, correct placement was achieved after reinsertion as observed by repeating intraoperative monitoring. In case No. 10, before reinsertion, the extended round window (eRW) opening was enlarged slightly, after which satisfactory placement was objectified with fluoroscopy and TIMmeasurement. Acquiring fluoroscopic images in this patient was complicated due to severely limited neck extension and rotation, causing difficulties in obtaining images in the correct view (modified Stenver's projection). 
TABLE 1. Details of patients with a tip foldover

\begin{tabular}{|c|c|c|c|c|c|c|c|c|c|}
\hline $\begin{array}{l}\text { Patient } \\
\text { No. }\end{array}$ & Gender & Age & CI Ear & $\begin{array}{c}\text { CI } \\
\text { Type }\end{array}$ & Approach & $\begin{array}{l}\text { Location of } \\
\text { Tip Foldover }\end{array}$ & Detected & Anatomy & $\begin{array}{l}\text { Etiology } \\
\text { of HL }\end{array}$ \\
\hline 9 & Male & 9 months & Right & CI632 & $\mathrm{CS}$ & E20 & Intraoperatively & Normal & DFNB1 \\
\hline 10 & Male & 65 & Right & CI632 & eRW & $\mathrm{E} 17 / 18$ & Intraoperatively & $\begin{array}{l}\text { Fenestral } \\
\text { otospongiosis }\end{array}$ & Unknown \\
\hline 12 & Female & 12 months & Right & CI632 & eRW & $\mathrm{E} 17 / 18$ & Intraoperatively & Normal & Congenital \\
\hline
\end{tabular}

CS indicates cochleostomy; eRW, extended round window; HL, Hearing loss.

The TIM-heatmap indicated foldovers at e20, e17/18, and 17/e18 in cases Nos, 9, 10, and 12 respectively, which was confirmed by fluoroscopy. Also, the location of the foldover estimated with the heatmap corresponded well with fluoroscopy (Fig. 2). The initial heatmap for case No. 12 further revealed an open circuit on stimulating e01, as can be observed in Figure 2 as a grey area. Cases Nos 4 and 6 both had open circuits at e16 and e23 , respectively.

UHR CT imaging 1 week postoperatively confirmed a satisfactory electrode placement in all patients and ruled out tip foldover $(\mathrm{N}=17$, Fig. 2C).

\section{DISCUSSION}

Correct placement of the electrode array is an important factor influencing outcome $(5,16)$. In this study, all three cases of electrode array tip foldover could be detected using TIM-measurement. Results of this current study further emphasize the value of intraoperative monitoring during cochlear implant surgery and indicate a promising role for TIM-measurements in detecting electrode array tip foldovers. As a result of peroperative recognition of the tip foldover, immediate reposition is possible and revision surgery can be prevented.

The results of the present study confirm that the SME has an increased risk of tip foldover compared with other electrode types $(6,9,12)$. However, the $12 \%$ tip foldover rate of found in our dataset is relatively high compared with what is described in other studies $(6,9,12)$. Because two of the three tip foldovers occurred in a child, a possible explanation could be the difficulty in optimally visualizing the round window in children due to the more lateral localization of the facial nerve (17). Because of this increased risk of tip foldover, intraoperative monitoring is imperative and, to-date, radiological imaging is the gold standard. This study showed the potential for TIM to detect tip foldovers in CI. Compared with imaging this has several advantages. Firstly, not all CI centers have access to intraoperative imaging. Secondly, imaging is more expensive, requires the presence of trained radiology personnel in the operating theater and is more time consuming. Thirdly, in using intraoperative fluoroscopy, there is an increased radiation exposure to both patient and theater staff. Lastly, the TIM-tool is intuitive and easy to interpret: with proper training, measurements can be performed and interpreted by an audiologist present in the operating room. In the future, slight adjustments to the software might also allow nonaudiological staff to perform the measurement.
In most of the participants, the heatmap showed higher transimpedances measured at apical contacts compared with basal contacts (Fig. 1). A possible explanation for this could be the presence of a current sink near the cochlear base (e.g., internal auditory meatus, cochlear aqueduct, or middle ear) and an increased resistivity near the apex due to reduction in cochlear diameter combined with an increased distance to the reference electrode. Although this could possibly lead to difficulties in discriminating a foldover of the single most apical electrode contact, the clinical relevance of such a limited foldover is uncertain and most probably insignificant.

Previously, two studies investigated the use of intraoperative objective measures for the detection of tip foldovers in CI recipients. Cosetti et al. (18) evaluated intraoperative electrode impedance (EI) measurement, NRT measurement, and plain film radiography for detecting tip foldovers in 277 patients. They concluded that of the three modalities, only radiological imaging influenced decision-making. In the four tip foldover cases they described, EIs and NRTs were normal. Grolman et al. (13) conducted a blind prospective trial to investigate the use of SOE measurements in 72 cochlear implantations and found that SOE was able to detect all four tip foldovers objectified using radiological imaging. In 2012, Vanpoucke et al. (15) proposed a method based on EFI which could possibly be used for intraoperative monitoring of cochlear implantation. TIM-measurement is a comparable objective measures test that is based on the analogous concept of visualizing spatial distribution of intracochlear potential.

As this study illustrates, it forms a promising modality for intraoperative detection of tip foldovers as it is available as a built-in tool in research software provided by the CI company, and only takes a few minutes to perform. As the surgeon is closing the cochleostomy and immobilizing the electrode array in the mastoid, the TIMprocessor can be applied and the measurement can be performed simultaneously. Compared with fluoroscopy, which usually takes 15 to 20 minutes of extra surgery time to perform, this is a great advantage. Using TIMmeasurements instead of intraoperative fluoroscopy therefore not only obviates the need of planning for fluoroscopy and having a radiology assistant on standby, but also leads to a reduction in surgery time and cost. Moreover, the frequency of doors opening during surgery is less and fewer people are needed in the operating room. In general, this is considered vital in implant surgery. At this stage, the decision about the presence/absence of a 
tip foldover is made independently by the expert clinician, either based on the information provided by fluoroscopy or by TIM. In our dataset, we feel that the informational value is comparable. It is conceivable that future detection algorithms, exclusively based on TIM, could automatically provide a reliable classification. Further clinical research into the sensitivity and specificity of this tool for detecting tip foldovers will be required before deciding to replace intraoperative imaging for this particular purpose altogether.

\section{CONCLUSION}

We conclude that TIM-measurement is a promising method for the intraoperative detection of electrode array tip foldovers with the potential to replace intraoperative fluoroscopy. The TIM-tool with intuitive heatmap display is easy to use, fast, and readily available to clinics using TIM-software in the operating theatre.

Acknowledgments: The authors would like to thank Dr. Filiep Vanpoucke (Head of Algorithms and Applications Cochlear Ltd.) for his advice and comments on the manuscript.

\section{REFERENCES}

1. Zanetti D, Nassif N, Redaelli de Zinis LO. Factors affecting residual hearing preservation in cochlear implantation. Acta Otolaryngol 2015;35:433-41.

2. Ramos-Macias A, R De Miguel A, Falcon-Gonzalez JC. Mechanisms of electrode fold-over in cochlear implant surgery when using a flexible and slim perimodiolar electrode array. Acta Otolaryngol 2017; 137:1129-35.

3. Zuniga MG, Rivas A, Hedley-Williams A, et al. Tip fold-over in cochlear implantation: case series. Otol Neurotol 2017;38:199206.

4. Holden LK, Finley CC, Firszt JB, et al. Factors affecting open-set word recognition in adults with cochlear implants. Ear Hear $2013 ; 34: 342-60$.
5. Wanna GB, Noble JH, Carlson ML, et al. Impact of electrode design and surgical approach on scalar location and cochlear implant outcomes. Laryngoscope 2014;124 (suppl):S1-7.

6. McJunkin JL, Durakovic N, Herzog J, Buchman CA. Early outcomes with a slim, modiolar cochlear implant electrode array. Otol Neurotol 2018;39:e28-33.

7. Cuda D, Murri A. Cochlear implantation with the nucleus slim modiolar electrode (CI532): a preliminary experience. Eur Arch Otorhinolaryngol 2017;274:4141-8.

8. Ramos-Macias A, Borkoski-Barreiro SA, Falcon-Gonzalez JC, Ramos-de Miguel A. Hearing preservation with the slim modiolar electrode nucleus CI532(R) cochlear implant: a preliminary experience. Audio Neurotol 2017;22:317-25.

9. Aschendorff A, Briggs R, Brademann G, et al. Clinical investigation of the Nucleus Slim Modiolar Electrode. Audio Neurotol 2017;22:169-79.

10. Gabrielpillai J, Burck I, Baumann U, Stover T, Helbig S. Incidence for tip foldover during cochlear implantation. Otol Neurotol 2018;39:1115-21.

11. Heutink F, Verbist BM, Mens LHM, Huinck WJ, Mylanus EAM. The evaluation of a slim perimodiolar electrode: surgical technique in relation to intracochlear position and cochlear implant outcomes. Eur Arch Otorhinolaryngol 2019;277:343-50.

12. Friedmann DR, Kamen E, Choudhury B, Roland JT Jr. Surgical experience and early outcomes with a slim perimodiolar electrode. Otol Neurotol 2019;40:e304-10.

13. Grolman W, Maat A, Verdam F, et al. Spread of excitation measurements for the detection of electrode array foldovers: a prospective study comparing 3-dimensional rotational $\mathrm{x}$-ray and intraoperative spread of excitation measurements. Otol Neurotol 2009;30:27-33.

14. de Rijk SR, Tam YC, Carlyon RP, Bance ML. Detection of extracochlear electrodes in cochlear implants with electric field imaging/transimpedance measurements: a Human Cadaver Study. Ear Hear 2020. doi:10.1097/AUD.0000000000000837.

15. Vanpoucke FJ, Boermans PP, Frijns JH. Assessing the placement of a cochlear electrode array by multidimensional scaling. IEEE Trans Biomed Eng 2012;59:307-10.

16. Lassig AA, Zwolan TA, Telian SA. Cochlear implant failures and revision. Otol Neurotol 2005;26:624-34.

17. Weiglein AH. Postnatal development of the facial canal an investigation based on cadaver dissections and computed tomography. Surg Radiol Anat 1996;18:115-23.

18. Cosetti MK, Troob SH, Latzman JM, Shapiro WH, Roland JT Jr, Waltzman SB. An evidence-based algorithm for intraoperative monitoring during cochlear implantation. Otol Neurotol 2012;33:169-76. 